\title{
Comportamento da pressão arterial sistêmica em pacientes submetidos a procedimentos cirúrgicos odontológicos
}

\section{Behavior of systemic blood pressure in patients submitted to dental surgery procedures}

\author{
Jefferson David Melo de Matos \\ Ana Larisse Carneiro Pereira** \\ Guilherme da Rocha Scalzer Lopes ${ }^{* * *}$ \\ Valdir Cabral Andrade ${ }^{* * * *}$ \\ Erick Gomes Perez $z^{* * * * *}$
}

\section{Resumo}

Objetivo: realizar uma revisão de literatura sobre a ocorrência de alterações significativas na pressão arterial sistêmica de pacientes submetidos a procedimentos cirúrgicos odontológicos. Materiais e método: esta revisão de literatura foi conduzida por meio das seguintes bases de dados: PubMed, SciELO e Google Scholar. As palavras-chave para a busca textual foram: cirurgia oral (oral surgery), hipertensão (hypertension), ansiedade (anxiety), odontologia (dentistry). Os critérios de inclusão foram: literatura sobre a temática em estudo, literatura dos últimos anos, literatura publicada em inglês ou português, estudos laboratoriais e clínicos e revisão sistemática. Os critérios de exclusão foram: revisão de literatura, carta ao editor, artigo de opinião, literatura duplicada em bases de dados e literatura que não abordasse as variáveis em estudo. Revisão de literatura: durante a prática clínica, o cirurgião-dentista lida rotineiramente com pacientes sistemicamente comprometidos. Entre eles, encontram-se os pacientes hipertensos, que necessitam de uma atenção diferenciada, devido a diversos fatores externos que podem influenciar sua condição sistêmica. Considerações finais: existe uma variação da pressão arterial durante todo procedimento cirúrgico odontológico, e o momento mais evidente dessa alteração é após a anestesia; essas variações, por sua vez, podem ser atribuídas à ansiedade e ao estresse causados pelo ato cirúrgico; a redução do estresse bem como o controle da ansiedade e do medo frente a um tratamento odontológico são benefícios no atendimento de pacientes hipertensos.

Palavras-chave: Ansiedade. Cirurgia oral. Hipertensão. Odontologia.

\section{Introdução}

A primeira aferição da pressão arterial (PA) foi realizada em uma égua, pelo inglês Stephen Hales (1677-1761). Quase um século após a descoberta, Jean Léonard Marie Poiseuille (1799-1869) melhorou o manômetro de Hales e criou o hemodinamômetro. Apesar de não ter uso prático, o hemodinamômetro foi um instrumento essencial, que serviu de base para o avanço de todos os aparelhos de aferir PA. Scipione Riva-Rocci é o responsável pelo marco, na era moderna, da aferição da PA. Em 1896, idealizou um aparelho composto de duas partes, uma destinada a exercer compressão sobre a artéria e outra a medir a pressão exercida. Chamou-o de angioparatlibômetro, sendo o responsável pelo fim da era das pesquisas de um método simples para avaliar a PA. ${ }^{1}$

O cirurgião russo Nikolai Korotkoff (1874-1920) foi o primeiro a aplicar a ausculta da artéria abaixo do manguito (em 1905), método que permitia a determinação da PA diastólica. O esfigmomanômetro de Riva-Rocci combinado com o uso do estetoscópio desenvolvido por Korotkoff, com apenas algumas modificações, continua sendo utilizado atualmente. ${ }^{2}$

Os principais métodos de aferição da PA reportados na literatura ocorrem por meio do método di-

Mestrando de Odontologia Restauradora, Departamento de Prótese Dentária, Universidade Estadual Paulista Júlio de Mesquita Filho, São José dos Campos, SP, Brasil.

Mestranda de Clínica Odontológica, Departamento de Odontologia, Universidade Federal do Rio Grande do Norte, Natal, RN, Brasil.

Doutorando de Odontologia Restauradora, Departamento de Prótese Dentária, Universidade Estadual Paulista Júlio de Mesquita Filho, São José dos Campos, Doutorando SP, Brasil.

**** Professor adjunto, Departamento de Odontologia, Universidade Federal de Juiz de Fora, Governador Valadares, MG, Brasil.

***** Professor de Cirurgia Oral e Maxilofacial, Departamento de Odontologia, Faculdade São Francisco de Assis, Santa Teresa, ES, Brasil. 
reto/invasivo e do método indireto/não invasivo. O método direto é considerado padrão-ouro de aferição da PA, por meio de um cateter intra-arterial. Entretanto, trata-se de uma técnica invasiva e impraticável em pacientes ambulatoriais. $\mathrm{O}$ método indireto ou não invasivo pode ser realizado pela técnica auscultatória (ausculta o aparecimento e o desaparecimento dos ruídos de Korotkoff, correspondentes às PAs sistólicas e diastólicas) ou oscilométrica (identifica o ponto de oscilação máxima correspondente a PA média e, por meio de algoritmos, as PAs sistólica e diastólica). Os métodos mais comuns para avaliar PA são aferição da PA casual no consultório, aferição ambulatorial da PA (Mapa) e aferição residencial da PA (MRPA). ${ }^{3}$

A Mapa avalia mais precisamente o risco de doença cardiovascular do que as aferições de PA realizadas durante consultas clínicas ou de consultório. A Mapa em 24 horas possibilita a observação contínua das mudanças na PA durante as atividades da vida diária, avaliando automaticamente em intervalos de tempo específicos e, portanto, permitindo avaliações mais precisas da PA. ${ }^{4} \mathrm{~A}$ MRPA é o método destinado a fazer registro da PA fora do consultório, realizada pelo próprio paciente ou por pessoa capacitada, com equipamento devidamente validado e calibrado, durante o período de vigília, por um longo período de tempo, obedecendo a um protocolo previamente estabelecido e normatizado. Este método permite realizar inúmeras aferições da PA e, assim, definir a PA de um indivíduo de forma mais fidedigna do que as aferições de consultório. Considera-se um exame anormal, quando as médias estiverem acima de 130 e/ou $85 \mathrm{mmHg}$ (grau de recomendação I - nível de evidência A). Não deve ser confundida com a automedida da PA, que é um registro não sistematizado e realizado a pedido do médico ou por decisão do próprio paciente. ${ }^{5,6}$

Para obter um controle da hipertensão arterial sistêmica (HAS), que acomete grande parte da população, é realizada a mensuração das pressões; esse passo é indispensável para o melhor diagnóstico, sendo de maior utilização entre os profissionais o esfigmomanômetro manual. ${ }^{7}$ A avaliação da alteração na PA precisa ser feita em aparelhos manuais, visto que estudos comprovam que a aferição automatizada não chega ao nível de precisão de um aparelho manual, tornando o modo manual um padrão de referência para precisão. ${ }^{8}$

O presente estudo tem por objetivo descrever, por meio de uma revisão de literatura, a ocorrência de alterações significativas na PA sistêmica de pacientes submetidos a procedimentos cirúrgicos odontológicos.

\section{Material e método}

Esta revisão de literatura foi conduzida por meio das seguintes bases de dados: PubMed, SciELO e Google Scholar. As palavras-chave para a busca textual foram: cirurgia oral (oral surgery), hipertensão (hypertension), ansiedade (anxiety), odontologia (dentistry). Os critérios de inclusão foram: literatura sobre a temática em estudo, literatura dos últimos anos, literatura publicada em inglês ou português, estudos laboratoriais e clínicos e revisão sistemática. Os critérios de exclusão foram: revisão de literatura, carta ao editor, artigo de opinião, literatura duplicada em bases de dados e literatura que não abordasse as variáveis em estudo.

\section{Revisão de literatura}

\section{Hipertensão arterial sistêmica}

A HAS é dividida em duas categorias principais: hipertensão primária ou essencial e hipertensão secundária. A hipertensão primária/essencial é de origem multifatorial, caracterizada pela falta de fatores causais identificáveis para PA elevada, ocorre em $90 \%$ a $95 \%$ de todos os casos de hipertensão. Os mecanismos associados a essas causas incluem: hereditariedade, mecanismos hemodinâmicos, neuronais, hormonais e renais. Três causas importantes são a sobrecarga de sal/volume, a ativação da renina-angiotensina-aldosterona, sistema nervoso central, e ativação do sistema nervoso simpático. ${ }^{9}$

A hipertensão secundária tem uma causa médica identificável e afeta de $5 \%$ a $10 \%$ dos adultos norte-americanos diagnosticados com hipertensão. Essas causas podem estar associadas a doenças vasculares, como coarctação da aorta, e doenças sistêmicas, como síndrome de Cushing, apneia obstrutiva do sono, disfunção medular da suprarrenal e disfunção hormonal, isto é, hiperaldosteronismo primário, feocromocitoma, hipertireoidismo, hiperparatireoidismo e hipotireoidismo, além do abuso de substâncias intorpecentes, consumo de álcool, uso de contraceptivos e doença renal crônica. ${ }^{10}$ Além das duas categorias principais abordadas, existem outras formas de manisfestação da HAS, como síndrome do jaleco branco (SJB) e hipertensão mascarada. ${ }^{11}$

A SJB, também denominada hipertensão do avental branco, é definida por PA elevada quando o paciente se encontra no consultório, diferentemente de quando está fora desse ambiente. Essa síndrome pode ser diagnósticada por vários metódos, a monitorização da PA em casa e em ambulatório durante 24 horas é um dele, sendo importantíssimo seu conhecimento por parte do cirurgião-dentista. ${ }^{12}$

Denomina-se hipertensão mascarada a situação em que há normotensão nas aferições de PA realizadas no consultório, embora a PA seja elevada nas 
medições fora desse ambiente, por Mapa durante o período de vigília ou por MRPA. ${ }^{13}$ Mais de $70 \%$ dos pacientes hipertensos estão cientes da doença, apenas entre 23 e $49 \%$ são tratados, e menos de $20 \%$ atingem controle da doença. A prevalência de HAS é em torno de 30 e $45 \%$ na população geral, apresentando aumento no envelhecimento. A hipertensão é considerada uma "assassina silenciosa", porque frequentemente afeta órgãos-alvo, como rins, coração, cérebro e olhos, antes do aparecimento de sintomas clínicos, aumentando a incidência de doença cardíaca coronariana, renal e cardíaca, e acidente vascular encefálico. ${ }^{14}$

As aferições das PAs sistólicas e diastólicas exigem uma definição dos limites de normalidade. De acordo com a última atualização da guidelines de hipertensão da sociedade americana de cardiologia, a PA passa a ser classificada da seguinte forma:

Quadro 1 - Classificação da HAS

\begin{tabular}{|c|c|}
\hline Normal & PAS $<120 \mathrm{mmHg}$ e PAD $<80 \mathrm{mmHg}$ \\
\hline Elevada & $\begin{array}{r}\text { PAS entre } 120 \text { e } 129 \mathrm{mmHg} \text { e PAD entre } \\
80 \text { e } 89 \mathrm{mmHg}\end{array}$ \\
\hline Hipertensão estágio I & $\begin{array}{r}\text { PAS entre } 130 \text { e } 139 \mathrm{mmHg} \text { ou PAD entre } \\
80 \text { e } 89 \mathrm{mmHg}\end{array}$ \\
\hline Hipertensão estágio II & $\begin{array}{r}\text { PAS acima ou igual a } 140 \mathrm{mmHg} \text { ou } \\
\text { PAD acima ou igual a } 90 \mathrm{mmHg}\end{array}$ \\
\hline
\end{tabular}

Fonte: adaptado de Whelton et al. ${ }^{15}$ (2017).

A fisiologia da hipertensão ocorre por meio de uma quantidade de sangue que o coração bombeia, também conhecida como débito cardíaco (DC) e resistência ao fluxo sanguíneo. Resulta de estreitamento das artérias inflexíveis, freqüência cardíaca elevada, aumento do volume sanguíneo ou contrações forçadas. A contração dos ventrículos é chamada de sístole, e a expansão dos ventrículos é chamada de diástole. A PA média é calculada multiplicando a PA diastólica por dois, somando a PA sistólica e dividindo tudo por três. A PA é controlada pelos rins por meio da liberação da enzima renina. A renina leva o angiotensinogênio à angiotensina 1 , que é convertida pela angiotensina enzima conversora para angiotensina 2 . A angiotensina 2 provoca vasoconstrição e resistência vascular elevada e estimula a libertação de aldosterona, uma enzima que aumenta a reabsorção de sódio no rim. No entanto, elevando a reabsorção de sódio, aumentam o volume sanguíneo e o nível da PA. ${ }^{16}$

$\mathrm{O}$ aumento da PA, por ser multifatorial, pode ser compreendido também por alterações hemodinâmicas, em que a PA é determinada pelo produto do DC e da resistência vascular periférica (RVP). Nos portadores de hipertensão arterial, existe um espectro de variação do DC com respostas concomitantes da RVP para um determinado nível de PA.
Essa diferença existe nas condições de repouso e em situações de estímulo. A contratilidade e o relaxamento do miocárdio, o volume sanguíneo circulante, o retorno venoso e a frequência cardíaca podem influenciar o DC. A RVP é determinada por vários mecanismos vasoconstrictores e vasodilatadores, como o sistema nervoso simpático, o sistema renina angiotensina e a modulação endotelial. A RVP depende também da espessura da parede das artérias, existindo uma potencialização ao estímulo vasoconstrictor nos vasos em que há espessamento de paredes. Em muitos pacientes com HAS, a elevação da PA é decorrente do aumento da RVP, já em outros a elevação do DC é responsável pela HAS. ${ }^{17}$

O sistema nervoso autônomo (SNS) é de grande importância para o controle da PA e pode estar alterado em pacientes com HAS. O inadequado funcionamento do SNS induz aumento do DC e RVP inapropriadamente elevada. Existem poucas controvérsias sobre a relação entre estimulação aguda do sistema nervoso simpático e desenvolvimento de HAS. Porém, há divergências se o SNS pode estar envolvido na elevação sustentada da PA. A angiotensina 2 atua na musculatura lisa dos vasos, produzindo constricção; no córtex adrenal, liberando aldosterona; na medula adrenal, liberando catecolaminas; em certas áreas do sistema nervoso central, iniciando a liberação de adrenalina para o cérebro e promovendo a ingesta de líquidos por estímulo no centro da sede no cérebro. Essas ações fisiologicamente atuam como uma defesa da PA, aumentando a RVP e a retenção de sódio e água. $\mathrm{O}$ feedback negativo dessa sequência homeostática fisiológica ocorre quando, na presença de excesso de angiotensina 2 , a liberação de renina é inibida. ${ }^{18}$

De acordo com Guedis et al. ${ }^{19}$ (2008), a SJB se relaciona à resposta pressórica do paciente na presença de um médico, cirurgião-dentista ou qualquer outro profissional da saúde. A SJB é uma condição clínica em que as PAs sistólica e diastólica são maiores que $20 \mathrm{mmHg}$ e $10 \mathrm{mmHg}$, respectivamente, em relação aos níveis obtidos pela medida da pressão de consultório, em comparação com aqueles registrados pela monitorização ambulatorial da PA ou pela monitorização residencial. A Mapa tem sido muito útil na avaliação da SJB, pois permite a medida intermitente da PA sem a presença do médico. Esse método apresenta melhor correlação com morbidade e mortalidade cardiovasculares do que a medida isolada de consultório. A SJB é mais frequente no sexo feminino e nas faixas etárias mais elevadas. Ainda não está esclarecida a implicação clínico-prognóstica da SJB. Estudos recentes demonstraram que população considerável de pacientes com diagnóstico de SJB se torna definitivamente hipertensa e, assim, atinge um risco cardiovascular intermediário entre aqueles com diagnóstico definitivo de HAS e os normotensos. É imprescindível que se saiba diferenciar a SJB de outras situações clínicas em que a PA encontra-se elevada, pois há tanto 
diferenças no prognóstico quanto a necessidade de tratamento específico, a fim de se ter o controle, com a monitorização ambulatorial ou residencial, da PA e melhorar a segurança no atendimento. ${ }^{19}$

Para a causa dessa síndrome, podem ser atribuídos conceitos por alerta, medo, ansiedade ou uma resposta condicional à situação incomum. A SJB é diferente do efeito jaleco branco, que se refere a uma PA elevada que pode ou não ocorrer no consultório. A prevalência da SJB durante as consultas é de aproximadamente $20 \%$, e a prevalência no cenário do consultório odontológico não foi estabelecida. $^{20}$

Em um estudo de Nicolosi et al. ${ }^{21}$ (2012), com o intuito de elucidar as alterações na PA e na frequência cardíaca de pacientes que passaram por tratamento odontológico sob uso de anestesia local, 82 pacientes foram avaliados antes do tratamento odontológico, no decorrer e depois deste, e os resultados demonstraram que houve aumento significativo da PA durante o procedimento odontológico, e os pacientes hipertensos apresentaram cinco vezes mais chances de ultrapassar os valores de 140/90mmHg durante o procedimento odontológico.

A alta taxa de prevalência faz com que a HAS afete de forma significativa o tratamento dos pacientes acometidos por essa doença. Segundo Umeizudike et al. ${ }^{22}$ (2013), em estudo realizado com 1910 pacientes de uma clínica odontológica na Nigéria, cerca de $39,9 \%$ eram hipertensos, e, destes, $42,1 \%$ não haviam recebido diagnóstico médico prévio.

\section{Histórico familiar de hipertensão}

A história familiar de uma população acometida pela HAS tem grande relevância, visto que essa doença cardiovascular tem fatores genéticos. Estudos demonstraram que essa condição genética chega a atingir $30 \%$ sobre a incidência de hipertensão. Para os que possuem histórico familiar de hipertensão, há mais chances de desenvolver HAS, então, torna-se importante que profissionais avaliem o histórico de saúde familiar do paciente. ${ }^{23}$

\section{Ansiedade}

A ansiedade pode ser caracterizada como um estado de estimulação central que envolve alterações metabólicas e neurotransmissores sinápticos; estes secretam substâncias que promovem a excitação ou a inibição do neurônio pós-sináptico. O sistema serotoninérgico fica responsável pela manifestação de ansiedade, enquanto o sistema noradrenérgico atua em situações de atenção ao ambiente na possibilidade de ameaça. O comportamento emocional se localiza no sistema límbico, que é interligado aos fenômenos de emoção, comportamento e controle do SNS, em que se destaca o papel do hipotálamo. ${ }^{24} \mathrm{O}$ SNS tem o poder de controlar as funções vegetativas do organismo e, para isso, utiliza vias simpática e parassimpática, sendo que a predominância da ação excitatória ocorre na via simpática. O sistema simpático reage na medula suprarrenal, fazendo com que esse órgão secrete adrenalina e noradrenalina. As secreções frente a um agente estressor produzirão efeitos a nível sistêmico, como: aumento da ativação orgânica, alteração térmica, aumento da PA e taquicardia, aumentando o metabolismo e desencadeando os sintomas de ansiedade. ${ }^{25}$

Durante a rotina clínica, a ansiedade vivida pelos pacientes é comumente observada. De acordo com Kakkar et al. ${ }^{26}$ (2016) em estudo realizado com 250 alunos, $42 \%$ das crianças com idades de 10 a 14 anos haviam vivenciado o medo de tratamento odontológico. Esse estado emocional pode desencadear situações que afetam o curso ideal do tratamento odontológico, como, por exemplo, a desistência por parte dos pacientes e a procura por um profissional apenas em casos extremos. ${ }^{27}$ Devido à ansiedade, há também a possibilidade de ocorrerem alterações hemodinâmicas durante procedimentos cirúrgicos, como alterações significativas na PA e na frequência cardíaca, como relatam Raocharernporn et al. ${ }^{28}$ (2017), em estudo que avaliou 27 pacientes saudáveis submetidos à cirurgia para extração de terceiros molares.

\section{Anestésicos locais}

Para reduzir a toxicidade sistêmica durante procedimentos odontológicos, elevar a duração e a eficácia da anestesia e promover uma hemostasia durante o procedimento cirúrgico, são usados vasocontritores em combinação com anestésicos locais, é o caso da lidocaína $2 \%$ com epinefrina 1:100.000. A epinefrina estimula os receptores $\alpha$ e $\beta$ do sistema adrenérgico em vários tecidos do corpo, e seus efeitos cardiovasculares, quando usada durante procedimentos odontológicos, têm sido explorados em muitos estudos. A anestesia local com epinefrina demonstrou aumentar a circulação dos níveis plasmáticos. Os níveis de epinefrina no plasma assim como a frequência cardíaca aumentaram em indivíduos jovens e saudáveis que receberam lidocaína com 20 ou $80 \mathrm{mg}$ de epinefrina. Esse efeito foi dependente da dose de interesse, a frequência cardíaca aumentou, e a PA diminuiu com o uso de epinefrina. O efeito aumentado da frequência cardíaca e a diminuição da PA ocorreram devido à estimulação do receptor $\beta 2$. A epinefrina usada em doses de 1 a 3 cartuchos de $1854 \mathrm{mg}$ não tem sido associada a efeitos adversos e, portanto, deve ser considerada segura, para uso na ausência de uma contraindicação. ${ }^{29} \mathrm{~A}$ dor durante o tratamento odontológico pode desencadear a liberação de catecolaminas endógenas, que podem levar a alterações hemodinâmicas, como aumento da PA e da frequência cardíaca, podendo até produzir arritmias, como relataram Abu-Mostafa et al. ${ }^{30}$ (2015). 
Para o cálculo das doses máximas de anestésicos, também chamado de cálculo de Clark, são necessárias algumas operações matemáticas. Primeiro, se a concentração do anestésico é de $2 \%$, significa que existem $20 \mathrm{mg}$ de anestésico em cada mililitro de solução. Uma solução de $3 \%$, então, terá 30 $\mathrm{mg}$ por mililitro. Cada tubete de $1,8 \mathrm{ml}$ de solução, multiplicando $20 \mathrm{mg}$ por 1,8 , tem $36 \mathrm{mg}$. Com esse valor, divide-se a quantidade máxima de sal anestésico que pode ser administrada para cada pessoa pela quantidade de sal em cada tubete, e obtém-se o número máximo de tubetes para aquele paciente, tendo o sal anestésico como limitante. Para o vasoconstritor, utiliza-se uma conta semelhante; em uma solução de 1:200.000, existe $1 \mathrm{~g}$ de vasoconstritor para cada $200.000 \mathrm{ml}$ de solução. Há, assim, $0,005 \mathrm{mg} / \mathrm{ml}$ de solução. Portanto, em uma solução de $1: 100.000$, há $0,01 \mathrm{mg} / \mathrm{ml}$. Cada tubete terá 0,009 $\mathrm{mg} / \mathrm{ml}$ para uma solução de 1:200.000 e $0,018 \mathrm{mg} /$ $\mathrm{ml}$ para uma de 1:100.000. Então, divide-se novamente o limite máximo de vasoconstritor pela quantidade presente em cada tubete, e tem-se o máximo de tubetes, tendo o vasoconstritor como limitante. $\mathrm{O}$ agente limitante para a definição do número máximo de tubetes pode ser tanto o sal anestésico quanto o vasoconstritor, dependendo de qual seja o menor valor. A dose do vasoconstritor epinefrina é de 0,2 mg e de 0,04 mg para cardiopatas. ${ }^{31}$

Para garantir maior segurança do procedimento a ser realizado, é importante estar atento ao equivalente metabólico da tarefa (MET) do paciente, que é definido como $3,5 \mathrm{ml}$ de oxigênio consumido por quilograma de massa corporal por minuto. Ele é um valor quantificador usado como teste da capacidade de realizar trabalho físico. ${ }^{32}$

Quadro 2 - Consumo energético em equivalentes metabólicos

\begin{tabular}{|c|l|}
\hline De 1 a 4 METs & $\begin{array}{l}\text { comer, vestir-se, usar o banheiro, caminhar dentro } \\
\text { de casa, caminhar a 3,2-4,8 km/h, realizar peque- } \\
\text { nas atividades domésticas }\end{array}$ \\
\hline De 4 a 10 METs & $\begin{array}{l}\text { subir um andar ou andar em subidas, caminhar a } \\
6,4 \mathrm{~km} / \mathrm{h} \text {, correr pequenas distâncias, limpar asso- } \\
\text { alhos ou móveis, fazer atividades físicas modera- } \\
\text { das, como dançar, jogar tênis em duplas }\end{array}$ \\
\hline$>10$ METs & $\begin{array}{l}\text { Realizar atividades físicas extenuantes, como nata- } \\
\text { ção, tênis, futebol, basquete }\end{array}$ \\
\hline
\end{tabular}

Fonte: adaptado de Novaes $^{33}$ (2006).

Por exemplo, se um paciente possuir capacidade de exercer de 1 a 4 METs, estará equivalendo a atividades como: comer, vestir-se, andar pela casa, lavar a louça. Já de 4 a 10 METs, a subir escadas, a caminhar a $6,4 \mathrm{~km} / \mathrm{h}$, a correr em curta distância, a jogar golfe. E acima de $10 \mathrm{METs}$, a praticar natação, tênis, futebol. Pacientes com capacidades de até 4 METs correm grande risco de complicações médicas, e aqueles de 10 METs ou mais possuem risco muito baixo. Um paciente que se encontra ansioso, com a PA $200 \mathrm{mmHg} / 115 \mathrm{mmHg}$, mas que pode exe- cutar 10 METs de trabalho, provavelmente não terá problemas com uma extração do tipo simples..$^{34}$

Com isso, tornam-se de suma importância a avaliação do aspecto de risco e o uso do METs antes de qualquer procedimento odontológico, precavendo-se de quaisquer intercorrências de complicações cardíacas. Para pacientes que fazem parte da classificação III e IV (Doença sistêmica grave e Distúrbio sistêmico grave que coloca em risco a vida do paciente, respectivamente) da American Society of Anesthesiologists (ASA) ou que têm capacidade de METs até 4, encoraja-se a dividir os procedimentos em vários quadrantes e a retomar as PAs após um quadrante da Odontologia. Uma técnica lenta com aspiração evita a injeção intravascular pelo operador e pode reduzir as reações adversas. A taxa sugerida para este procedimento é de $1 \mathrm{ml} / \mathrm{min} .^{35}$

Quadro 3 - Classificação do estado físico

\begin{tabular}{|c|l|}
\hline Classificação & \multicolumn{1}{|c|}{ Definição } \\
\hline ASA I & $\begin{array}{l}\text { Não há alterações fisiológicas ou orgânicas, e o } \\
\text { processo patológico responsável pela cirurgia não } \\
\text { causa problemas sistêmicos. }\end{array}$ \\
\hline ASA II & $\begin{array}{l}\text { Há alteração sistêmica leve ou moderada rela- } \\
\text { cionada com patologia cirúrgica ou enfermidade } \\
\text { geral. }\end{array}$ \\
\hline ASA III & $\begin{array}{l}\text { Há alteração sistêmica intensa relacionada com } \\
\text { patologia cirúrgica ou enfermidade geral. }\end{array}$ \\
\hline ASA IV & $\begin{array}{l}\text { Há distúrbio sistêmico grave que coloca em risco } \\
\text { a vida do paciente. }\end{array}$ \\
\hline ASA V & $\begin{array}{l}\text { Paciente moribundo que não é esperado que so- } \\
\text { breviva sem a operação. }\end{array}$ \\
\hline ASA VI & $\begin{array}{l}\text { Paciente com morte cerebral declarada, cujos ór- } \\
\text { gãos estão sendo removidos com propósitos de } \\
\text { doação. }\end{array}$ \\
\hline
\end{tabular}

Fonte: American Society of Anesthesiologists ${ }^{35}$ (2012).

Com o objetivo de comparar os parâmetros cardiovasculares, a eficácia anestésica e o grau de desconforto durante a injeção de 2 soluções anestésicas de lidocaína a $2 \%$ associada a concentrações diferentes de epinefrina, estudo demonstrou que não houve diferenças estatisticamente significativas entre os valores de PA sistólica (Friedman, $p=0,33$ ), PA diastólica (Friedman, $p=0,1505$ ), frequência cardíaca (Friedman, $p=0,9464$ ) e saturação de oxigênio (Friedman, $p=0,9297$ ), considerando cada anestésico local durante e após a anestesia. ${ }^{36}$

$\mathrm{O}$ aumento na pressão diastólica, utilizando a epinefrina, pode se relacionar ao aumento na concentração sanguínea, ocorrendo, assim, estímulo também dos receptores $\alpha$ com vasoconstrição na musculatura esquelética. Pacientes com hipertensão controlada, no estágio I ou II da doença, que usam medicação anti-hipertensiva, têm tolerado doses bem pequenas de anestésico com epinefrina e podem passar por tratamento odontológico. Já hipertensos no estágio III podem ser submetidos 
apenas a procedimentos que não sejam invasivos em caso de emergências, evitando qualquer tipo de procedimento cirúrgico. ${ }^{37}$

\section{Obesidade}

A obesidade é uma doença crônica não transmissível, geralmente assintomática, podendo atingir o padrão de doença mórbida, classe grave III, quando o índice de massa corporal (IMC) atinge $40 \mathrm{~kg} / \mathrm{m} 2$. As doenças crônicas não transmissíveis no cenário da obesidade, cujas consequências são multifatoriais, geram principalmente repercussões cardiovasculares e hipertensivas. ${ }^{38}$

Atualmente, o diagnóstico preciso de obesidade não se restringe apenas ao cálculo de IMC. A medida da circunferência da cintura e a relação cintura-quadril também fornecem dados, como peso de músculos e gorduras não enquadrado pelo método do IMC. ${ }^{39}$ Medidas de circunferência da cintura acima de $102 \mathrm{~cm}$ e $88 \mathrm{~cm}$ em homens e mulheres, respectivamente, apresentam alto risco para várias doenças associadas à obesidade, principalmente risco de desenvolver doenças cardiovasculares. ${ }^{40}$

O estilo de vida moderno inclui fatores como sedentarismo, maus hábitos alimentares, uso de tabaco, consumo excessivo de álcool e obesidade; esses aspectos influenciam muito o desenvolvimento da hipertensão. Dong et al. ${ }^{41}$ (2013) investigaram as tendências da PA e a influência do IMC em crianças, com base em pesquisas nacionais chinesas entre 1985 e 2010 com mais de um milhão de crianças em idade escolar. A análise revelou a prevalência da PA sistólica elevada: que diminuiu de 5,1\% para $3,5 \%$ em meninos, e de $5,5 \%$ para $2,5 \%$ em meninas, entre 1985 e 2005; e aumentou para 4,9\% e 3,5\% em 2010, respectivamente. A prevalência de obesidade aumentou de $0 \%$ para 3,4\% em meninos e 0,9\% em meninas. Quanto à PA diastólica, um padrão semelhante foi observado. Depois de diminuir por 20 anos, os níveis de PA em crianças chinesas começaram a subir. $\mathrm{O}$ estudo sugeriu que deve-se investigar com urgência as mudanças seculares em outros fatores de risco e os preventivos da PA elevada, para melhorar os resultados da saúde cardiovascular na população chinesa.

Em uma associação entre nível sérico de adiponectina e hipertensão de início recente e a contribuição relativa da obesidade e de baixos níveis séricos de adiponectina para o desenvolvimento de hipertensão em homens e mulheres normotensos, revelou-se que o ganho de peso está relacionado com elevação da PA, visto que a hipertensão arterial é três vezes mais frequente em obesos do que em não obesos, e o risco eleva-se com o aumento do peso. ${ }^{42}$

\section{Sedentarismo}

A atividade sedentária é definida como gasto de energia referente à menos de 1,5 MET. Sendo que $1 \mathrm{MET}$ representa 3,5 $\mathrm{ml}$ de oxigênio consumido por quilo de massa corporal por minuto e inclui atividades como sentar, ver televisão, andar, entre outras. No nível global, há hipóteses de que 55\% a $70 \%$ das atividades realizadas diariamente sejam do tipo sedentária. Em nível nacional, os chilenos experimentaram mudanças importantes em seus estilos de vida durante as últimas três décadas, e atualmente $19,8 \%$ da população é fisicamente inativa. Além disso, 35,9\% da população ficam mais de 4 horas por dia sentada, aumentando as chances de prejudicar sua saúde cardiovascular. ${ }^{43}$

O sedentarismo está interligado ao estilo de vida e é fator predisponente para risco de HAS. Atualmente, há um grande número de pessoas que não possui o hábito de fazer atividade física, tornando-se, assim, sedentária. Os adultos passam cerca de $57 \%$ de suas horas de trabalho sem realizar atividade física. É preciso que a população desenvolva um estilo de vida melhor, para que seja controlado e prevenido o quadro de HAS, por meio de um plano de atividades. ${ }^{44}$

\section{Tabagismo}

O tabagismo e a PA elevada são dois fatores de risco importantes para doenças cardiovasculares. O tabagismo é uma exposição ambiental com forte preditor para o desenvolvimento da HAS. ${ }^{45}$

O uso de cigarro é uma das principais causas de morte evitáveis no mundo, e, de acordo com a Organização Mundial de Saúde, estima-se que até 2030 o tabaco causará cerca de 8 milhões de mortes a cada ano. Ao analisar os efeitos de interação da intensidade do tabagismo (por exemplo, a quantidade de cigarros fumados por dia) e variantes genéticas na PAS em três diferentes grupos de exposição ao tabagismo: fumantes atuais, ex-fumantes e nunca fumantes, usando três diferentes covariantes de interação da quantidade de cigarros fumados por dia (10,15 e 20 cigarros por dia), descobriu-se que os fumantes atuais revelaram os sinais mais fortes para a interação do tabagismo com genes do cromossomo 6 (SASH1) e do cromossomo 3 (KLHL6), e foi associado o aumento da PA com exposição a curto prazo, e a diminuição da PA, com maior exposição. ${ }^{46}$

\section{Etilismo}

O consumo do álcool afeta tanto áreas social e psicológica quanto a área da saúde, sendo um grande fator de risco para doenças cardiovasculares como a HAS. ${ }^{47} \mathrm{O}$ mecanismo que leva o consumo de álcool a ser fator de risco para HAS é a ativação do álcool do sistema renina-angiotensina-aldosterona, afetando a função endotelial vascular, com inibição dos efeitos vasodilatadores e aumento da PA. ${ }^{48}$

Há relações de que os quadros de HAS ligados à dependência do álcool sejam mais prevalentes em homens do que em mulheres. ${ }^{49}$ Deve-se levar em 
consideração que esse fator de risco para hipertensão pode se potencializar, quando associado a sedentarismo, obesidade e uso de tabaco. ${ }^{50}$

\section{Discussão}

Uma preocupação importante durante a prática clínica entre os cirurgiões-dentistas é o uso de anestésicos locais com ou sem vasoconstritores, visto que o procedimento pode aumentar de forma abrupta a PA do paciente. Silvestre et al. ${ }^{51}$ (2011) não observaram alterações hemodinâmicas entre os grupos que usaram vasoconstritores e os que não usaram.

No que diz respeito ao anestésico, quando observado se apresentava elevação na PA dos pacientes, independentemente de esses serem hipertensos ou não, não foram encontradas relações estatisticamente significantes nos cruzamentos das alterações na PA com os anestésicos. Isso corrobora com os estudos de Goulart et al. ${ }^{52}$ (2012), Ogunlewe et al. ${ }^{53}$ (2011), Gungormus e Buyukkurt ${ }^{54}$ (2003), em que não houve alteração significativa da $\mathrm{PA}$ em relação ao tipo de solução anestésica empregada.

Acerca do uso da epinefrina em pacientes hipertensos, Perusse et al. ${ }^{55}$ (1992) e Carvalho et al. ${ }^{56}$ (2013) recomendam que a dose máxima segura seja de 2 tubetes de lidocaina $2 \%$ com epinefrina $1: 100.000$. Niwa et al. ${ }^{57}$ (2001) e Bader et al. ${ }^{58}$ (2002) revelaram um pequeno aumento da $\mathrm{PA}$, quando associado ao uso da epinefrina em pacientes hipertensos controlados; porém, Bronzo ${ }^{59}$ (2005), Perusse et al. ${ }^{55}$ (1992), Soares et al. ${ }^{60}$ (2006) e Cáceres et al. ${ }^{61}$ (2008) recomendam o uso dos vasoconstritores sem comprometimentos ao paciente, desde que a dose máxima recomendada seja respeitada. Niwa et al. ${ }^{57}$ (2001) enfatizam que o vasoconstrictor promove uma anestesia adequeada, duradoura, além de promover uma homeostasia, levando à redução a dor do paciente e à liberação de catecolaminas.

Alemany-Martínez et al. ${ }^{62}$ (2008), Santos ${ }^{63}$ (2012) e Erazo et al. ${ }^{64}$ (2014) afirmam que a atenuação do estresse associada a ansiolíticos ou sedação, quando bem indicados, produz efeitos benéficos na redução da resposta cardiovascular relacionada à ansiedade pré-operatória do paciente, embora a primeira técnica a ser utilizada nesses casos seja o controle comportamental do paciente mediado pelo dentista, como explicações prévias sobre como o procedimento odontológico vai ser realizado, melhorando a ansiedade e permitindo uma melhor condução do procedimento.

Medeiros et al. ${ }^{65}$ (2013), Carvalho et al. ${ }^{56}$ (2013), Loggia et al. ${ }^{66}$ (2008) e Tanno e Marcondes ${ }^{67}$ (2002) relataram a ação fisiológica da ansiedade na alteração da PA e afirmaram que o medo e a ansiedade influenciam na aferição da pressão. A alteração da PA pode ser explicada pelo estresse acarretado no ambiente ambulatorial, fazendo com que sejam liberados os principais mediadores da reação de estresse: as catecolaminas, liberadas pelo sistema nervoso simpático e pela medula da glândula suprarrenal, e os glicocorticoides, liberados pelo córtex da suprarrenal, em que ambos propiciam eventos celulares que viabilizam mudanças adaptativas em células e tecidos, com o papel de proteger o organismo e garantir a sua sobrevivência. Em nível de sistema nervoso central, a liberação endógena de adrenalina produz um efeito excitatório. Em resposta a essas ações, ocorre o aumento da PA, além de eventual alteração de potencialização da anestesia local.

Oliveira et al. ${ }^{37}$ (2010) e Goulart et al. ${ }^{52}$ (2012) afirmaram que a ansiedade, o medo e a dor não foram capazes de alterar significativamente a PA, contrariando os autores anteriormente citados. Isso pode ser explicado pelo tipo de procedimento realizado, já que o estudo de Oliveira et al. ${ }^{37}$ (2010) abordou tratamento endodôntico considerado menos estressante que extrações dentárias. Outra explicação está no fato de os autores terem trabalhado com uma amostra diferente, em termos quantitativos, da utilizada neste estudo $(\mathrm{n}=135)$.

No estudo de Goulart et al. ${ }^{52}$ (2012), utilizou-se uma amostra de 60. Outros fatores estudados, como sexo, etnia, alcoolismo e antecedentes familiares, não demonstraram nesta pesquisa relevância estatisticamente significativa $(p>0,050)$, quando relacionados com o estado de HAS, apesar de sua correlação comprovada em diversos estudos; portanto, estudos mais detalhados devem ser realizados para uma melhor comprovação dessa relação.

Alterações nas PAs sistólica e diastólica são observadas em pacientes antes e no decorrer de procedimentos clínicos odontológicos. Essas alterações podem estar associadas a dor, estresse decorrente do medo e ansiedade durante o procedimento cirúrgico, também a fatores individuais, como idade, hipertensão, experiência prévia traumática em tratamento odontológico, resposta psicológica, maus hábitos alimentares, sedentarismo, IMC e uso de tabaco. Em grande parte desses pacientes submetidos a procedimentos odontológicos observa-se elevação da $\mathrm{PA}$ durante todo o procedimento clínico realizado.

\section{Considerações finais}

Pode se concluir a partir deste estudo que existe uma variação da PA durante todo procedimento cirúrgico odontológico, e o momento que essa alteração torna-se mais evidente é após a anestesia, corroborando com os dados da literatura. Essas variações podem ser atribuídas à ansiedade e ao estresse causados pelo ato cirúrgico; a redução do estresse bem como o controle da ansiedade e do medo frente a um tratamento odontológico são benéficos no atendimento de pacientes hipertensos. Vale ressaltar que a aferição da PA pré-operatória e sua monitorização durante toda a extensão do procedimento cirúrgico 
são de fundamental importância para que as melhores condutas sejam tomadas.

\section{Abstract}

Objective: the objective of this study was to describe through a review of the literature on the occurrence of significant changes in the systemic arterial pressure of patients submitted to dental surgical procedures. Materials and method: this literature review was conducted using the databases: PubMed, SciELO and Google Scholar. The keywords for the literature search were: Oral surgery; Hypertension; Anxiety; Dentistry. The inclusion criteria were: papers that covers the subject under study, papers of the last years, papers in English and Portuguese language, laboratory studies, clinical studies and systematic review. The exclusion criteria were: literature review, letter to the editor, opinion article, papers duplicated in databases and literature that did not address the variables under study. Literature review: in the clinical practice, the dental surgeon routinely treats systemically compromised patients. Among them are the hypertensive patients who need a differentiated attention due to several external factors that can influence their systemic health. Final considerations: there are a variation of the arterial pressure throughout the dental surgical procedure, the moment that this change becomes more evident is after the anesthesia; these variations in turn can be attributed to the anxiety and stress caused by the surgical act; the reduction of surgical stress as well as the control of anxiety and fear of dental treatment are benefits in the care of hypertensive patients.

Keywords: Anxiety. Oral surgery. Hypertension. Dentistry.

\section{Referências}

1. Booth J. A short history of blood pressure measurement. Proc R Soc Med 1977 Nov; 70(11):793-9.

2. Van Gijn J, Gijselhart JP. [Riva-Rocci and blood pressure]. Ned Tijdschr Geneeskd 2013; 157(3):A5536.

3. Hogan J, Radhakrishnan J. The assessment and importance of hypertension in the dental setting. Dent Clin North Am 2012 Oct; 56(4):731-45.

4. Kim SK, Bae JH, Nah DY, Lee DW, Hwang TY, Lee KS. Frequency and related factors of masked hypertension at a worksite in Korea. J Prev Med Public Health 2011; 44(3):1319.

5. Pickering TG, Miller NH, Ogedegbe G, Krakoff LR, Artinian NT, Goff D, et al. Call to action on use and reimbursement for home blood pressure monitoring: a joint scientific statement from the American Heart Association, American Society Of Hypertension, and Preventive Cardiovascular Nurses Association. Hypertension 2008 ;52(1):10-29.

6. De Monitorização Residencial IDB. III Diretrizes Brasileiras de Monitorização Residencial da Pressão Arterial (MRPA). Rev Bras Hipertens 2011; 18(1):18-25.

7. Zahid H, Amin A, Amin E, Waheed S, Asad A, Faheem A, et al. Prevalence and Predictors of Use of Home Sphygmomanometers Among Hypertensive Patients. Cureus 2017 11; 9(4):e1155.
8. Mirdamadi A, Etebari M. Comparison of manual versus automated blood pressure measurement in intensive care unit, coronary care unit, and emergency room. ARYA Atheroscler 2017; 13(1):29-34.

9. Southerland JH, Gill DG, Gangula PR, Halpern LR, Cardona CY, Mouton CP. Dental management in patients with hypertension: challenges and solutions. Clin Cosmet Investig Dent 2016 17(8):111-20.

10. Hogan J, Radhakrishnan J. The assessment and importance of hypertension in the dental setting. Dent Clin North Am 2012; 56(4):731-45.

11. Rimoldi SF, Scherrer U, Messerli FH. Secondary arterial hypertension: when, who, and how to screen? Eur Heart J 2014 May 14; 35(19):1245-54.

12. Hogan J, Radhakrishnan J. The assessment and importance of hypertension in the dental setting. Dent Clin North Am 2012 Oct; 56(4):731-45.

13. Imai $Y$, Obara $T$, Asamaya $K$, Ohkubo T. The reason why home blood pressure measurements are preferred over clinic or ambulatory blood pressure in Japan. Hypertens Res 2013 Aug; 36(8):661-72.

14. Matsuura $\mathrm{H}$. The systemic management of cardiovascular risk patients in dentistry. Anesth Pain Control Dent. 1993 Winter; 2(1):49-61.

15. Whelton PK, Carey RM, Aronow WS, Casey-Jr DE, Collins KJ, Dennison-Himmelfarb C, et al. ACC/AHA/AAPA/ABC/ ACPM/AGS/APhA/ASH/ASPC/NMA/PCNA guideline for the prevention, detection, evaluation, and management of high blood pressure in adults: a report of the American College of Cardiology/American Heart Association Task Force on Clinical Practice Guidelines. JACC Basic Transl Sci, 24430, 2017.

16. Bavitz JB. Dental management of patients with hypertension. Dental Clinics 2006; 50(4):547-62.

17. Sanjuliani AF. Fisiopatologia da hipertensão arterial: conceitos teóricos úteis para a prática clínica. Rev SOCERJ 2002; 15(4):210-8.

18. Sealey JE, Laragh JH. The renin-angiotensinaldosterone system for normal regulation of blood pressure and sodium and potassium homeostasis. In: Laragh JH, Brenner BM, editors. Hypertension: Pathophisiology, Diagnosis and Management. New York: Raven Press; 1995; 27(12):1287-317.

19. Guedis AG, Sousa BDBD, Marques CF, Piedra DPS, Braga JCMS, Cardoso MLG, et al. Hipertensão do avental branco e sua importância de diagnóstico. Rev Bras Hipertens 2008; 15(1):46-50.

20. Popescu SM, Scrieciu M, Mercu $\square$ V, $\square$ uculina M, Dasc $\square$ lu I. Hypertensive patients and their management in dentistry. ISRN Hypertension 2013; 2013(410740):1-8.

21. Nicolosi L, Lewin P, Winter G, Medina F, Carballo J, Martinez C, et al. Comparative study of changes in arterial pressure and heart rate during dental treatment under local anesthesia in hypertensive patients versus normotensive patients. Acta Odontol Latinoam 2012; 25(3):318-23.

22. Umeizudike KA, Ayanbadejo PO, Umeizudike TI, Isiekwe GI, Savage KO. Relevance of routine blood pressure assessment among dental patients in Lagos, Nigeria. J Contemp Dent Pract 2013; 1,14(6):1145-50.

23. Ong P, Pirozzolo G, Athanasiadis A, Sechtem U. Epicardial Coronary Spasm in Women With Angina Pectoris and Unobstructed Coronary Arteries Is Linked With a Positive Family History: An Observational Study. Clin Ther 2018 Aug 16; pii:S0149-2918(18)30320-5. 
24. Nascimento ER, Maia AC, Nardi AE, Silva AC. Sexual dysfunction in arterial hypertension women: the role of depression and anxiety. J Affect Disord 2015 Aug 1; 181:96-100.

25. Doganer YC, Aydogan U, Yesil HU, Rohrer JE, Williams MD, Agerter DC. Does the trait anxiety affect the dental fear? Braz Oral Res 2017 May 4; 31:e36.

26. Kakkar M, Wahi A, Thakkar R, Vohra I, Shukla AK. Prevalence of dental anxiety in 10-14 years old children and its implications. J Dent Anesth Pain Med 2016 Sep; 16(3):199-202.

27. Yagiela JA, Haymore TL. Management of the hypertensive dental patient. J Calif Dent Assoc 2007 Jan; 35(1):51-9.

28. Raocharernporn S, Boonsiriseth K, Khanijou M, Wongsirichat N. Hemodynamic changes and pain perception-related anxiety after experiencing an impacted-tooth removal: clinical practice outcome. J Dent Anesth Pain Med 2017 Jun; 17(2):105-11.

29. Ritter AV. High blood pressure and oral health. J Esthet Restor Dent 2007; 19(2):125-6.

30. Abu-Mostafa N, Al-Showaikhat F, Al-Shubbar F, Al-Zawad $\mathrm{K}, \mathrm{Al}-Z a w a d \mathrm{~F}$. Hemodynamic changes following injection of local anesthetics with different concentrations of epinephrine during simple tooth extraction: a prospective randomized clinical trial. J Clin Exp Dent 2015 Oct 1; 7(4):e471-6.

31. Santaella GM. Soluções anestésicas locais: uma revisão de literatura. Rep UFSC 2011; 1-61.

32. Saravane D, Feve B, Frances Y, Corruble E, Lancon C, Chanson $\mathrm{P}$, et al. [Drawing up guidelines for the attendance of physical health of patients with severe mental illness]. Encephale 2009 Sep; 35(4):330-9.

33. Novaes MV. Avaliação e preparo pré-operatório: classificação do estado físico. Medicina perioperatória. Rio de Janeiro: SAERJ; 2006.

34. Newcomer JW. Metabolic syndrome and mental illness. Am J Manag Care 2007 Nov; 13(7 Suppl):S170-7. Review. Erratum in: Am J Manag Care. 2008 Feb; 14(2):76.

35. American Society of Anesthesiologists. 1995-2012. American Society of Anesthesiologist (ASA). Sept. 10. 2012.

36. De Hert M, Dekker JM, Wood D, Kahl KG, Holt RI, Möller HJ. Cardiovascular disease and diabetes in people with severe mental illness position statement from the European Psychiatric Association (EPA), supported by the European Association for the Study of Diabetes (EASD) and the European Society of Cardiology (ESC). Eur Psychiatry 2009; 24(6):412-24

37. Oliveira PC, Zanetta-Barbosa D, Souza HJ, Batista JD, Ranali J, Costa MD, et al. Avaliação do nível de ansiedade e dor de pacientes em urgências endodônticas e sua influência sobre parâmetros cardiovasculares. Brazilian Dental Science $2010 ; 10(4): 70-5$.

38. Matsuda M, Shimomura I. Increased oxidative stress in obesity: implications for metabolic syndrome, diabetes, hypertension, dyslipidemia, atherosclerosis, and cancer. Obes Res Clin Pract 2013 Sep-Oct; 7(5):e330-41.

39. Wannmacher L. Obesidade como fator de risco para morbidade e mortalidade: evidências sobre o manejo com medidas não medicamentosas. Uso Racion Medicam Fundam em condutas Ter e nos macroprocessos da Assist Farm 2016; 1(7):110.

40. Traissac P, Pradeilles R, El Ati J, Aounallah-Skhiri H, Eymard-Duvernay S, Gartner A, et al. Abdominal vs. overall obesity among women in a nutrition transition context: geographic and socio-economic patterns of abdominal-only obesity in Tunisia. Popul Health Metr 2015 Jan 24; 13:1.
41. Dong B, Wang HJ, Wang Z, Liu JS, Ma J. Trends in blood pressure and body mass index among Chinese children and adolescents from 2005 to 2010. Am J Hypertens 2013 Aug; 26(8):997-1004.

42. Jung DH, Kim JY, Kim JK, Koh SB, Park JK, Ahn SV. Relative contribution of obesity and serum adiponectin to the development of hypertension. Diabetes Res Clin Pract 2014 Jan; 103(1):51-6.

43. Leiva AM, Martínez MA, Cristi-Montero C, Salas C, Ramírez-Campillo R, Díaz Martínez X, et al. [Sedentary lifestyle is associated with metabolic and cardiovascular risk factors independent of physical activity]. Rev Med Chil 2017 Apr; 145(4):458-67.

44. Gao Y, Xie X, Wang SX, Li H, Tang HZ, Zhang J, et al. Effects of sedentary occupations on type 2 diabetes and hypertension in different ethnic groups in North West China. Diab Vasc Dis Res 2017 Jul; 14(4):372-5.

45. Basson J, Sung YJ, Fuentes LL, Schwander K, Cupples LA, Rao DC. Influence of Smoking Status and Intensity on Discovery of Blood Pressure Loci Through Gene-Smoking Interactions. Genet Epidemiol 2015 Sep; 39(6):480-8.

46. Sung YJ, de Las Fuentes L, Schwander KL, Simino J, Rao DC. Gene-smoking interactions identify several novel blood pressure loci in the Framingham Heart Study. Am J Hypertens 2015 Mar; 28(3):343-54.

47. Dong Z, Lou P, Zhang P, Chen P, Qiao C, Li T. [Relationship between alcohol dependence and new detected hypertension in adult residents of Xuzhou city]. Zhonghua Xin Xue Guan Bing Za Zhi 2015 Dec; 43(12):1083-7.

48. Ji A, Lou P, Dong Z, Xu C, Zhang P, Chang G, et al. The prevalence of alcohol dependence and its association with hypertension: a population-based cross-sectional study4 in Xuzhou city, China. BMC Public Health 2018 Mar 16; 18(1):364.

49. Wakabayashi M, McKetin R, Banwell C, Yiengprugsawan V, Kelly M, Seubsman SA, et al. Alcohol consumption patterns in Thailand and their relationship with non-communicable disease. BMC Public Health 2015 Dec 24; 15:1297.

50. Bellis MA, Hughes K, Nicholls J, Sheron N, Gilmore I, Jones L. The alcohol harm paradox: using a national survey to explore how alcohol may disproportionately impact health in deprived individuals. BMC Public Health 2016 Feb 18; 16:111.

51. Silvestre FJ, Salvador-Martínez I, Bautista D, SilvestreRangil, J. Clinical study of hemodynamic changes during extraction in controlled hypertensive patients. Med Oral Patol Oral Cir Bucal 2011; 16(3):e354-8.

52. Goulart JCF, Pinheiro MD, Rodrigues RV, Santos FDSAD, Martins AT, Scannavino FLF. Influence of anxiety on blood pressure and heart rate during dental treatment. Revista Odonto Ciência 2012; 27(1):31-5.

53. Ogunlewe MO, James O, Ajuluchukwu JN, Ladeinde AL, Adeyemo WL, Gbotolorun OM. Evaluation of haemodynamic changes in hypertensive patients during tooth extraction under local anaesthesia. West Indian Medical Journal 2011; 60(1):91-5

54. Gungormus M, Buyukkurt MC. The evaluation of the changes in blood pressure and pulse rate of hypertensive patients during tooth extraction. Acta medica Austriaca 2003; 30(5):127-9.

55. Perusse R, Goulet JP, Turcotte JY. Contraindications to vasoconstrictors in dentistry: part II. Hyperthyroidism, diabetes, sulfite sensitivity, cortico-dependent asthma, and pheochromocytoma. Oral surgery, oral medicine, and oral pathology 1992; 74(5):687-91. 
56. Carvalho B, Fritzen EL, Parodes AG, Santos RB, Gedoz L. O emprego dos anestésicos locais em Odontologia: revisão de literatura. Rev Bras Odontol 2013; 70(2):178-81.

57. Niwa H, Sugimura M, Satoh Y, Tanimoto A. Cardiovascular response to epinephrine-containing local anesthesia in patients with cardiovascular disease. Oral Surgery, Oral Medicine, Oral Pathology, Oral Radiology and Endodontics 2001; 92(6):610-6.

58. Bader JD, Bonito AJ, Shugars DA. A systematic review of cardiovascular effects of epinephrine on hypertensive dental patients. Oral Surgery, Oral Medicine, Oral Pathology, Oral Radiology and Endodontics 2002; 93(6):647-53.

59. Bronzo ALA. Procedimentos odontológicos em pacientes hipertensos com ou sem o uso de anestésico local prilocaína associada ou não ao vasoconstritor felipressina [Doctoral dissertation]. São Paulo: Universidade de São Paulo; 2005.

60. Soares RG, Salles AA, Irala DLE, Limongi O. Como escolher um adequado anestésico local para as diferentes situações na clínica odontológica diária?. Rev Sul-Brasileira Odontol $2006 ; 3(1): 35-40$.

61. Cáceres MTF, Ludovice ACP, Brito FSD, Darrieux FC, Neves RS, Scanavacca MI, et al. Efeito de anestésicos locais com e sem vasoconstritor em pacientes com arritmias ventriculares. Arq Bras Cardio 2008; 91(3):142-7.

62. Alemany-Martínez A, Valmaseda-Castellón E, Berini-Aytés L, Gay-Escoda C. Hemodynamic changes during the surgical removal of lower third molars. J Maxillofac Oral Surg 2008; 66(3):453-61.

63. Santos DP. Controle da ansiedade odontológica: estudo comparativo entre a sedação oral com midazolam e a sedação consciente com a mistura de óxido nitroso e oxigênio em pacientes submetidos à extração de terceiros molares inferiores [Doctoral dissertation]. São Paulo: Universidade de São Paulo; 2012.

64. Erazo RM, Ronda AH, Alcayaga RG. Ansiedad dental: evaluación y tratamiento. Avan Odont 2014; 30(1):39-46.

65. Medeiros LDA, Ramiro FMS, Lima CAA, Souza LMDA, Fortes TMV, Groppo FC. Avaliação do grau de ansiedade dos pacientes antes de cirurgias orais menores. Rev Odont UNESP 2013; 42(5):357-63.

66. Loggia ML, Schweinhardt P, Villemure C, Bushnell MC. Effects of psychological state on pain perception in the dental environment. J Can Dent Assoc 2008 Sep; 74(7):651-6.

67. Tanno AP, Marcondes FK. Estresse, ciclo reprodutivo e sensibilidade cardíaca às catecolaminas. Rev Bras Cisc Farm $2002 ; 38(3): 273-89$.

Endereço para correspondência:

Jefferson David Melo de Matos

Avenida Francisco Engenheiro José Longo, 555,

Jardim São Dimas

12245-000, São José dos Campos, SP, Brasil

Telefones: (+55) (27) 99958-1702/

(+55) (88) 99603-9595

E-mail: matosjefferson19@gmail.com 